\title{
Alterations in adipocyte glucose transporter GLUT4 and circulating adiponectin and visfatin in rat adjuvant induced arthritis
}

\author{
Jana Jurčovičová ${ }^{1,2}$, Andrea Štofková2 ${ }^{2}$ Martina Škurlová ${ }^{2}$ Miroslava Baculíková1 ${ }^{1}$ Štefan \\ Zórad $^{1}$ and Mária Stančíková ${ }^{3}$ \\ ${ }^{1}$ Institute of Experimental Endocrinology, Slovak Academy of Sciences, Bratislava, Slovakia \\ E-mail: jana.jurcovicova@savba.sk \\ ${ }^{2}$ Department of Normal, Pathological and Clinical Physiology, Third Faculty of Medicine, Charles University, Prague, \\ Czech Republic \\ ${ }^{3}$ Institute for Rheumatic Diseases, Pieštany, Slovakia
}

\begin{abstract}
Rheumatoid arthritis in humans brings about impaired insulin sensitivity and glucose tolerance. Since adipose tissue plays a role in glucose homeostasis, we evaluated the size of adipocytes, the amount of glucose transporter type 4 (GLUT4) in adipocyte plasma membranes, and circulating insulin, glucose, and adipokines affecting glucose metabolism, resistin, adiponectin and visfatin during experimental adjuvant arthritis (AA) in male Lewis rats. AA was induced by a single injection of complete Freund's adjuvans. Adipocyte diameter was assessed microscopically, GLUT4 was measured by Western blotting. Plasma insulin, adiponectin, visfatin were quantitated by RIA, and resistin by ELISA. Arthritic rats showed cachexia, reduced adipocyte size, and downregulated membrane GLUT4 (4065 \pm 962 vs. $9911 \pm 680$ arb. units of optic density, $p<0.01$ ), reduced plasma adiponectin $(1.956 \pm 0.10 v s .3 .16 \pm 0.22 \mu \mathrm{g} / \mathrm{ml}, p<0.001)$, and enhanced visfatin $(1.84 \pm 1.05 v s$. $1.24 \pm 0.1 \mathrm{ng} / \mathrm{ml}, p<0.01)$. Plasma glucose and insulin were unaltered, as were the resistin levels. Conclusion: AA induced cachexia results in reduction of adipocyte size, and paradoxically also in downregulation of GLUT4 in adipocyte membranes. This is supposed to be functionally related to the reduced adiponectin levels. The upregulated visfatin in rat arthritis is a novel finding, and it confirms its role in autoimmunity across the species.
\end{abstract}

Key words: Adjuvant arthritis - Adipocytes - GLUT4 - Visfatin - Adiponectin

\section{Introduction}

Numerous clinical studies evidenced metabolic consequences of rheumatoid arthritis (RA), often leading to insulin resistance and metabolic syndrome in these patients (Dessein et al. 2006; Sidiropoulos et al. 2008). It has been known that the systemic inflammatory process characteristic of RA brings about changes in a whole array of adipokines that may further affect the severity of the disease.

Resistin has been first found to induce insulin resistance and glucose intolerance in obesity (Steppan et al. 2001;

Correspondence to: Jana Jurčovičová, Institute of Experimental Endocrinology, Slovak Academy of Sciences, Vlárska 3, 83306 Bratislava, Slovakia

E-mail: jana.jurcovicova@savba.sk
Guzik et al. 2006). More recently a local proinflammatory role has been ascribed to resistin, since it stimulated interleukin- 6 and tumor necrosis factor- $\alpha$ in human leukocytes, its enhanced local joint production in patients with RA was observed and its intra-articular administration to mice induced inflammation (Bokarewa et al. 2005). Moreover, in severe RA higher resistin levels occurred in the circulation (Senolt et al. 2007).

Adiponectin is known to enhance insulin sensitivity in the tissue. It promotes insulin regulated glucose transport by its direct action on the GLUT4 transporter expression and translocation into plasma membrane to ameliorate insulin resistance (Fu et al. 2005). Adiponectin has been found in the synovial adipocytes and fibroblasts of RA patients. In the joint it stimulated interleukin- 6 production and matrix degrading enzymes suggesting its local proinflammatory activity (Ehling et al. 2006). Adiponectin was also enhanced 
in the circulation and synovial fluid of RA patients (Otero et al. 2006; Senolt et al. 2006).

Visfatin (nicotinamide phosphoribosyltransferase - NAMPT), was originally described as pre-B-cell colonyenhancing factor (Samal et al. 1994), and later as insulinmimetic adipokine secreted from visceral fat (Fukuhara et al. 2005). Subsequently visfatin/NAMPT was shown to act as extracellular NAD biosynthetic enzyme critical for glucosestimulated insulin secretion in pancreatic $\beta$-cells (Revollo et al. 2007). Recent study revealed that visfatin can improve insulin sensitivity by enhancing insulin receptor substrate- 1 phosphorylation, as well as by up-regulation of peroxisome proliferator-activated receptor- $\gamma$ in fat tissue (Sun et al. 2009). Visfatin has been qualified as a proinflammatory agent with matrix degrading activity in the synovial tissue. At the same time its levels in circulation and in synovial fluid have been enhances in patients with RA (Otero et al. 2006; Brentano et al. 2007).

Rat adjuvant arthritis (AA) represents a widely used experimental model for RA. A single subcutaneous injection of a suspension of heat killed Mycobacterium butyricum in mineral oil develops polyarthritis. The disease exhibits a dynamic progression with an initial, preclinical phase followed within about 10 days by acute clinical phase which reaches peak intensity around day 20 . It is characterized by a number of changes occurring in human RA. In Lewis arthritic rats from day 12 onwards the loss of appetite is accompanied by reduction in plasma levels of leptin, adiponectin, and also by downregulation of the negative reactant of acute phase protein, albumin (Stofkova et al. 2009). Similarly, in the same strain of rats from day 21 through 28 of AA, an overall oxidative stress has been observed (Mihalova et al. 2007; Strosova et al. 2008). In this model, on day 18 of AA we evaluated the size of adipocytes, the amount of GLUT4 in adipocyte plasma membranes in male Lewis rats to ascertain possible effects of polyarthritis on glucose transport. At the same time we assessed the circulating levels of glucose, insulin, as well as the adipokines involved in insulin metabolism and arthritis, resistin adiponectin, and visfatin.

\section{Materials and Methods}

\section{Animals}

Male Lewis 8-weeks old rats (Charles River, Germany) were housed four per cage in an animal room in the Department of Normal, Pathological and Clinical Physiology, Third Faculty of Medicine, Charles University, Prague. They were treated according to the national law of the Czech Republic on the use of laboratory animals, No. $167 / 1993$. AA was induced to rats by a single subcuta- neous injection of $100 \mu \mathrm{l}$ of complete Freund's adjuvant (cFA) into the base tail. Rats were decapitated on day 18 after cFA injection along with intact controls (8 rats per group). Trunk blood was collected into EDTA, centrifuged and plasma was stored at $-30^{\circ} \mathrm{C}$ until assayed. Epididymal fat was taken to measure adipocyte size and GLUT4 in adipocyte plasma membranes. We analyzed epididymal fat only, because the amount of any other fat is extremely limited in arthritic animals, and is not sufficient for analyses.

\section{Diameter of adipocytes and GLUT4 measurement}

One part of epididymal fat was used for adipocyte size determination. Adipocytes were isolated by collagenase digestion (Pinterova et al. 2001) and the fat cell size was assessed microscopically. The cells were photographed using a camera (Canon Digital Power Shot S40) attached to the microscope and the cell diameter was calculated. Resulting diameter is an average value of at least 100 cells from each adipocyte suspension. The rest of the epididymal fat was homogenized, and plasma membranes were isolated as detailed previously (Baculikova et al. 2008). For GLUT4 Western blot, $20 \mu \mathrm{g}$ solubilized fat tissue membranes were separated by electrophoresis on $12 \%$ TRIS-glycine polyacrylamide gel and than electrotransferred to Hybond C Extra membrane (Amersham Bioscience, Buckinghamshire, UK). After blocking for $1 \mathrm{~h}$ in TBS with 5\% milk, the membrane was incubated overnight at $4^{\circ} \mathrm{C}$ with primary rabbit antiGLUT4 antibody (Abcam, Cambridge, UK) diluted $1: 2500$ in $10 \mathrm{mmol} / \mathrm{l} \mathrm{Tris} \mathrm{-} 150 \mathrm{mmol} / \mathrm{l} \mathrm{NaCl}$ buffer, $\mathrm{pH} 7.4$ (TBS) with $0.2 \%$ Igepal (Sigma, St. Louis, USA). The membrane was washed in TBS-Igepal buffer and than incubated with horseradish peroxidase-conjugated secondary antibody (goat anti-rabit IgG, $1: 15$ 000, Sigma, St. Louis, USA) for $1 \mathrm{~h}$ at room temperature. After washing the membrane was exposed to chemiluminescence reagent (Amersham Bioscience, Buckinghamshire, UK) and exposed to X-ray film. The chemiluminescence signal was acquired by densitometric scanning.

\section{Analyses}

Albumin was determined by spectrophotometric method using SYS 1 kit (BM/Hitachi, Boehringer Mannheim, Germany) on Hitachi 911 automatic biochemical analyzer (Boehringer Mannheim, Germany). Insulin, adiponectin and visfatin concentrations were determined by the respective RIAs (Linco Research, USA), resistin by ELISA (BioVendor, Czech Republic) or glucose using auto-analyzer Hitachi 911(Hitachi, Japan). The data were analyzed by unpaired Student's $t$-test. Statistical significance is considered at $p<$ 0.05 . 


\section{Results}

The hind paw volumes were significantly enlarged in AA rats that correlated with overall cachexia typical for the developed phase of AA. As expected, the epididymal fat stores were significantly smaller in arthritic rats $(422 \pm 70 \mathrm{mg} v s .1184 \pm$ $105 \mathrm{mg}$ in controls, $p<0.001)$, and similarly body mass was reduced $(201 \pm 25.9 \mathrm{~g} v s .325 \pm 2.9 \mathrm{~g}$ in controls, $p<0.001)$. AA markedly inhibited the production of albumin levels in rat. It occurred as a result of enhanced acute phase proteins synthesis by the liver secondary to the activation of hepatic cells by inflammatory cytokines (Figure 1).

The body and fat mass loss resulted in a reduction of adipocyte diameter. Furthermore the fat cells clearly showed downregulation of GLUT4 translocation into the cell membranes. Inflammation did not affect either glucose, or insulin plasma levels at unrestricted food intake (Figure 2). In the Figure 3 are depicted values for circulating adipokines. Plasma resistin levels, in spite of its described role in autoimmune inflammation remained unchanged under our conditions. However, circulating adiponectin involved in GLUT4 regulation was reduced along with the reduction of fat mass. On the other hand, the levels of circulating visfatin were enhanced in arthritic rats that inversely correlated to the reduced fat mass.

A

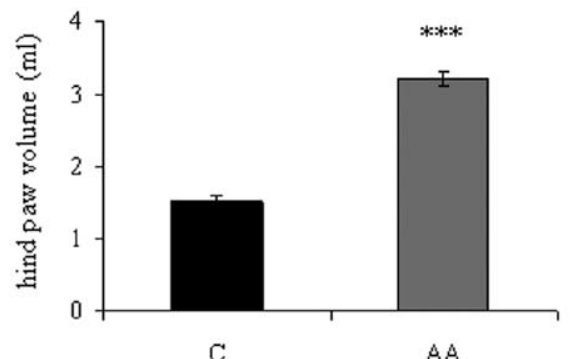

$\mathrm{C}$

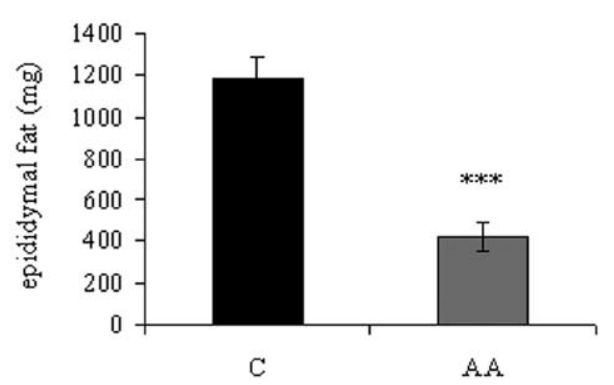

\section{Discussion}

Here we described reduced adipocyte diameter, and a clearcut downregulation of GLUT4 in the adipocyte plasma membranes in the fully developed phase of AA characterized by joint swelling, body and fat mass loss, and reduction of albumin production. At the same time we found unaffected basal glucose and insulin levels, unchanged circulating resistin, but reduced adiponectin and enhanced visfatin levels.

Physiological reduction of fat mass is usually associated with an increase in insulin sensitivity and upregulation of GLUT4 in adipocytes. For example, $40 \%$ caloric restriction reversed insulin resistance in pinealectomized rats by enhancing GLUT4 content in adipocytes and its translocation to the plasma membranes (Zanquetta et al. 2003). Similarly in fatty rats caloric restriction improved glucose utilization by upreguletion of adipocyte GLUT4 expression (Park et al. 2005). Under the conditions of clinically manifested AA the loss of appetite occurs, that is manifested by reduced food consumption by about $40 \%$, and consequently by body and fat mass loss (Stofkova et al. 2009). The inflammatory reduction of GLUT4 in adipocyte plasma membranes, that we observed, is a specific phenomenon that can not be attributed to lowered caloric intake, but mirrors the adipokine imbalance during the disease. Since we confirmed this

B

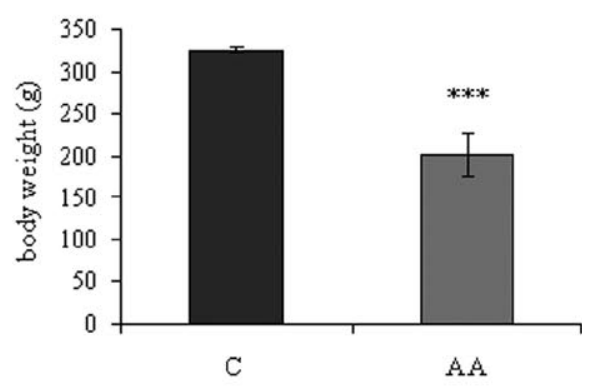

D

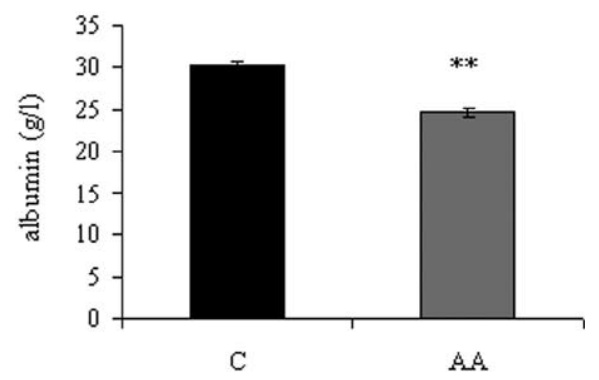

Figure 1. Clinical signs of adjuvant arthritis represented by paw edema (A), loss of body weight (B), and epididymal fat (C). The enhanced production of acute phase proteins is manifested by decreased albumin production (D). Each column represents mean of 7-8 animals fed ad libitum \pm S.E.M. ${ }^{* *} p<0.01 ;{ }^{* * *} p<0.001$; C, intact control rats; AA, arthritic rats on day 18 of the disease. 
A

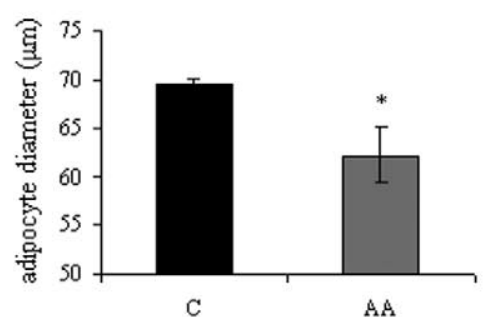

C

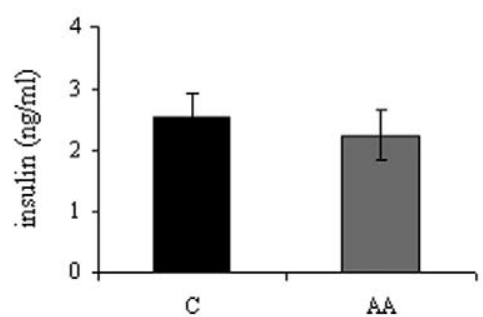

B

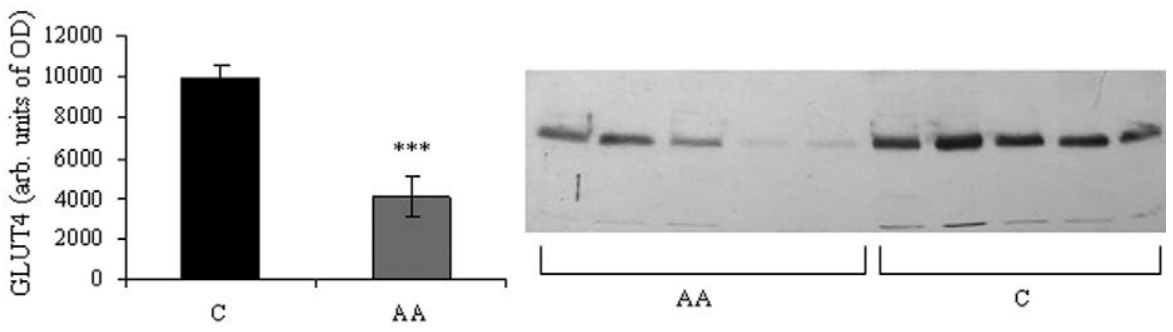

$\mathrm{D}$

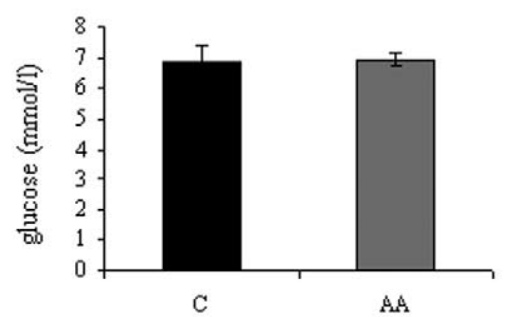

Figure 2. Diameter of adipocytes (A), and the amount of GLUT4 (B), in adipocyte plasma membranes in normal and arthritic rats. The bands stand for representative Western blot of GLUT4 in 5 healthy and 5 arthritic animals. In the lower part are depicted plasma insulin (C) and glucose (D) levels in rats. Each column represents mean of 7-8 animals fed ad libitum \pm S.E.M. ${ }^{\star} p<0.05 ;{ }^{* * *} p<0.001$; $\mathrm{C}$, intact control rats; AA, arthritic rats on day 18 of the disease; OD, optic density.

A

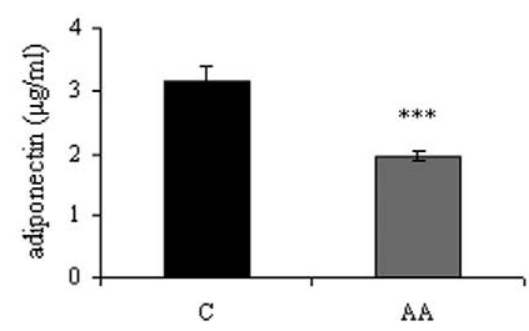

B

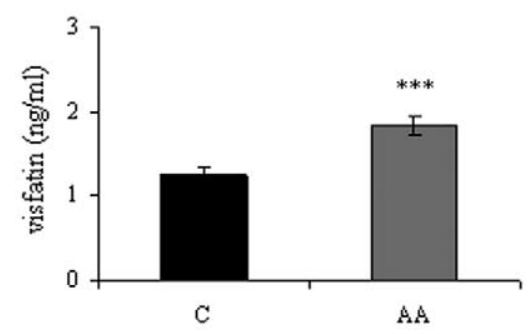

C

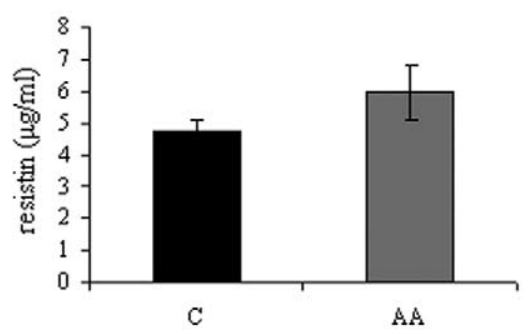

Figure 3. Plasma adiponectin (A), visfatin (B), and resistin (C) in normal and arthritic rats. Each column represents mean of 7-8 animals fed ad libitum \pm S.E.M. ${ }^{* *} p<0.001$; , intact control rats; AA, arthritic rats on day 18 of the disease.

finding also in the Long Evans arthritic rats (unpublished), we assume that it is a general feature accompanying autoimmune experimental arthritis.

Our result on the reduction of circulating adiponectin levels during AA confirm the recent finding of Haruna et al. (2007), Martin et al. (2008), and our own observation (Stofkova et al.2009) in the clinical phase of AA, and suggests that this may be involved in the downregulation of GLUT4 in adipocytes. Any interpretation of reduced adiponectin plasma levels related to the severity of the disease can not be drawn, because the decisive role plays its intraarticular production. Regarding the unaltered resistin levels in our experiment, we assume that in spite of its defined local proinflammatory activity, circulating resistin is not an important factor in the development of AA. In this study we described for the first time enhanced visfatin/NAMPT levels in arthritic rats. Visfatin/NAMPT has been shown to be upregulated in the activated immune cells (Rongvaux et al. 2002), and recently Busso et al. (2008) showed its enhanced levels in the circulation and in the inflamed paws in collagen-induced arthritis (CIA) in mice as well. These authors clearly demonstrated its role in the pathology of the disease, 
since its pharmacological blockade improved the clinical features of CIA. Whether enhanced visfatin levels acts also to compensate insulin insensivity due to lack of GLUT4, and maintains unaltered basal insulin and glucose during autoimmunity, warrants further study.

In conclusion, AA-induced cachexia results in reduction of adipocyte size, and paradoxically also in downregulation of GLUT4 in adipocytes. This is supposed to be related to the reduced adiponectin levels. The enhanced visfatin levels in rat $\mathrm{AA}$ is a novel finding demonstrating that this occurs across the species, and rat AA provides a relevant model for further study of its mechanisms.

Acknowledgements. This work was supported by GACR 305/06/ 0427, VZ 0021620816 and APVV 21-055205 grants.

\section{References}

Baculikova M., Fiala R., Jezova D., Macho L., Zorad S. (2008): Rats with monosodium glutamate-induced obesity and insulin resistance exhibit low expression of Gai2 G-protein. Gen. Physiol. Biophys. 27, 222-226

Bokarewa M., Nagaev I., Dahlberg L., Smith U., Tarkowski A. (2005): Resistin, an adipokine with potent proinflammatory properties. J. Immunol. 174, 5789-5795

Brentano F., Schorr O., Ospelt C., Stanczyk J., Gay R. E., Gay S., Kyburz D. (2007): Pre-B cell colony-enhancing factor/ visfatin, a new marker of inflammation in rheumatoid arthritis with proinflammatory and matrix-degrading activities. Arthritis Rheum. 56, 2829-2839; doi:10.1002/ art.22833

Busso N., Karababa M., Nobile M., Rolaz A., Van Gool F., Galli M., Leo O., So A., De Smedt T. (2008): Pharmacological inhibition of nicotinamide phosphoribosyltransferase/visfatin enzymatic activity identifies a new inflammatory pathway linked to NAD. PLoS ONE. 21, e2267; doi:10.1371/journal.pone.0002267

Dessein P. H., Tobias M., Veller M. G. (2006): Metabolic syndrome and subclinical atherosclerosis in rheumatoid arthritis. J. Rheumatol. 33, 2425-2432

Ehling A., Schaffler A., Herfarth H., Tarner I. H., Anders S., Distler O., Paul G., Distler J., Gay S., Scholmerich J., Neumann E., Muller-Ladner U. (2006): The potential of adiponectin in driving arthritis. J. Immunol. 176, 4468-4475

Fu Y., Luo N., Klein R. L., Garvey W. T. (2005): Adiponectin promotes adipocyte differenciation, insulin sensitivity, and lipid accumulation. J. Lipid Res. 46, 1369-1379; doi:10.1194/jlr.M400373-JLR200

Fukuhara A., Matsuda M., Nishizawa M., Segawa K., Tanaka M., Kishimoto K., Matsuki Y., Murakami M., Ichisaka T., Murakami H., Watanabe E., Takagi T., Akiyoshi M., Ohtsubo T., Kihara S., Yamashita S. Makishima M., Funahashi T., Yamanaka S., Hiramatsu R., Matsuzawa Y., Shimomura I. (2005): Visfatin: a protein secreted by visceral fat that mimics the effects of insulin. Science 307, 426-430; doi:10.1126/science.1097243
Guzik T. J., Mangalat D., Korbut R. (2006): Adipocytokines - novel link between inflammation and vascular function? J. Physiol. Pharmacol. 57, 505-528

Haruna Y., Morita Y., Yada T., Satoh M., Fox D. A., Kashihara N. (2007): Fluvastatin reverses endothelial dysfunction and increased vascular oxidative stress in rat adjuvant-induced arthritis. Arthritis Rheum. 56, 1827-1835; doi:10.1002/ art.22632

Martin A. I., Castillero E., Granado M., Lopez-Menduina M., Villanua M. A., Lopez-Calderon A. (2008): Adipose tissue loss in adjuvant arthritis is associated with a decrease in lipogenesis, but not with an enhance in lipolysis. J. Endocrinol. 197, 111-119; doi:10.1677/JOE-07-0491

Mihalova D., Ponist S., Kucharska J., Komendova D., Bauerova K. (2007): Total antioxidant status - systemic marker of oxidative stress in adjuvant arthritis. Chem. Letters 101, 225-226

Otero M., Lago R., Gomez R., Lago F., Dieguez C., GomezReino J. J., Gualillo O. (2006): Changes in plasma levels of fat-derived hormones adiponectin, leptin, resistin and visfatin in patients with rheumatoid arthritis. Ann. Rheum. Dis. 65, 1198-1201; doi:10.1136/ ard.2005.046540

Park S. Y., Choi G. H., Choi H. I., Ryu J., Jung C. Y., Lee W. (2005): Caloric restriction improves whole-body glucose disposal and insulin resistance in association with the increased adipocyte-specific GLUT4 expression in Otsuka LongEvans Tokushima fatty rats. Arch. Biochem. Biophys. 436, 276-284; doi:10.1016/j.abb.2005.01.010

Pinterova L., Zelezna B., Fickova M., Macho L., Krizanova O., Jezova D, Zorad S. (2001): Elevated AT1 receptor protein but lower angiotensin II-binding in adipose tissue of rats with monosodium glutamate-induced obesity. Horm. Metab. Res. 33, 708-712; doi:10.1055/s-2001-19132

Revollo J. R., Korner A., Mills K. F., Satoh A., Wang T., Garten A., Dasgupta B., Sasaki Y., Wolberger C., Townsend R. R., Milbrandt J., Kiess W., Imai S. (2007): Nampt/PBEF/ visfatin regulates insulin secretion in $\beta$ cells as a systemic NAD biosynthetic enzyme. Cell Metab. 6, 363-375; doi:10.1016/j.cmet.2007.09.003

Rongvaux A., Shea R. J., Mulks M.H., Gigot D., Urbain J., Leo O., Andris F. (2002): Pre-B-cell colony-enhancing factor, whose expression is up-regulated in activated lymphocytes, is a nicotinamide phosphoribosyltransferase, a cytosolic enzyme involved in NAD biosynthesis. Eur. J. Immunol. 32, 3225-3234; doi:10.1002/1521-4141(200211)32:11<3225:: AID-IMMU3225>3.0.CO;2-L

Samal B., Sun Y., Stearns G., Xie C., Suggs S., McNiece I. (1994): Cloning and characterization of the cDNA encoding a novel human pre-B-cell colony-enhancing factor. Mol. Cell. Biol. 14, 1431-1437

Senolt L., Pavelka K., Housa D., Haluzik M. (2006): Increased adiponectin is negatively linked to the local inflammatory process in patients with rheumatoid arthritis. Cytokine 35, 247-252; doi:10.1016/j.cyto.2006.09.002

Senolt L., Housa D., Vernerova Z., Jirasek T., Svobodova R., Veigl D., Anderlova K., Müller-Ladner U., Pavelka K., Haluzík M. (2007): Resistin in rheumatoid arthritis synovial tissue, 
synovial fluid, and serum. Ann. Rheum. Dis. 66, 458-463; doi:10.1136/ard.2006.054734

Sidiropoulos P. I., Karvounaris S. A., Boumpas D. T. (2008): Metabolic syndrome in rheumatic diseases: epidemiology, pathophysiology, and clinical implications. Arthritis Res. Ther. 10, 207; doi:10.1186/ar2397

Steppan C. M., Bailey S. T., Bhat S., Brown E. J., Banerjee R. R., Wright C. M., Patel H. R., Ahima R. S., Lazar M. A. (2001): The hormone resistin links obesity to diabetes. Nature 409, 292-293; doi:10.1038/35053000

Stofkova A., Haluzik M., Zelezna B., Kiss A., Skurlova M., Lacinova Z., Jurcovicova J. (2009): Enhanced expressions of mRNA for neuropeptide $\mathrm{Y}$ and interleukin 1 beta in hypothalamic arcuate nuclei during adjuvant arthritis-induced anorexia in Lewis rats. Neuroimmunomodulation 16, 377-384; doi: $10.1159 / 000228912$

Strosova M., Tomaskova I., Ponist S., Bauerova K., Karlovska J., Spickett C. M., Horakova L. (2008): Oxidative im- pairment of plasma and skeletal muscle sarcoplasmic reticulum in rats with adjuvant arthritis - effects of pyridoindole antioxidants. Neuro Endocrinol. Lett. 29, 706-711

Sun Q., Li L., Li R., Yang M., Liu H., Nowicki M. J., Zong H., Xu J., Yang G. (2009): Overexpression of visfatin/ $\mathrm{PBEF} /$ Nampt alters whole-body insulin sensitivity and lipid profile in rats. Ann. Med. 41, 1-11; doi:10.1080/ 07853890902729760

Zanquetta M. M., Seraphim P. M., Sumida D. H., Cipolla-Neto J., Machado U. F. (2003): Caloric restriction reduces pinealectomy-induced insulin resistance by improving GLUT4 gene expression and its translocation to the plasma membrane. J. Pineal Res. 35, 141-148; doi:10.1034/j.1600-079X.2003.00067.x

Received: April 13, 2009

Final version accepted: October 19, 2009 